\title{
EUS-guided gallbladder drainage: a learning curve modified by technical progress
}

\section{(ㄷ)(1)}

Authors

Amy Tyberg ${ }^{1}$, Kopal Jha ${ }^{2}$, Shawn Shah ${ }^{3}$, Prashant Kedia ${ }^{4}$, Monica Gaidhane ${ }^{1}$, Michel Kahaleh $^{1}$

Institutions

1 Division of Gastroenterology, Rutgers Robert Wood Johnson Medical Center, New Brunswick, New Jersey, United States

2 Cornell University, Ithaca, New York, United States

3 Weill Cornell Medicine, New York, New York, United Stats

4 Methodist Health System, Dallas, Texas, United States

submitted 23.4.2019

accepted after revision $\quad 6.8 .2019$

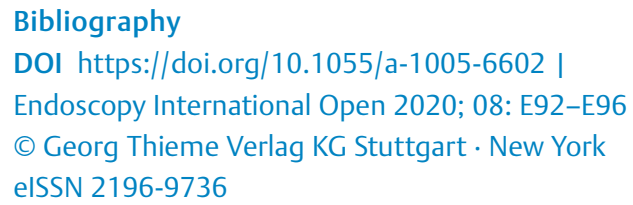

Corresponding author

Michel Kahaleh, MD, AGAF, FACG, FASGE, Clinical Director of Gastroenterology, Chief of Endoscopy, Director Pancreas Program, Rutgers, The State University of New Jersey, Robert Wood Johnson University Hospital, 1 RWJ Place, MEB 464, New Brunswick, NJ 08901

Fax: +1-732-235-5537

mkahaleh@gmail.com

\section{ABSTRACT}

Introduction Endoscopic ultrasound-guided gallbladder drainage (EUS-GBD) is an efficacious and safe option for patients who cannot undergo cholecystectomy. It is a techni- cally challenging procedure, requiring skills in EUS, and ERCP. The aim of this study was to define the learning curve for EUS-GBD.

Patients and methods Consecutive patients undergoing EUS-GBD by a single operator were included from a prospective registry over 5 years. Demographics, procedure information, post-procedure follow-up data, and information on adverse events were collected. Non-linear regression and CUSUM analyses were conducted for the learning curve. Clinical success was defined as resolution of cholecystitis post-procedure.

Results Forty-eight patients were included (58\% male, mean age 76 years). Twenty patients ( $42 \%$ ) had malignant cholecystitis. Most patients had lumen-apposing metal stents (LAMS) (15 mm, $\mathrm{n}=29,60 \% ; 10 \mathrm{~mm}, \mathrm{n}=8,7 \%$ ). The remaining patients had FCSEMS $(n=9,19 \%)$ or plastic stents alone $(n=2,4 \%)$. Clinical success was achieved in 36 ( $86 \%$ ) of patients. Of the remaining 12,7 were lost to follow-up and 5 had persistent cholecystitis. 9 patients (19\%) had adverse events including bleeding $(n=4)$, liver abscesses $(n=2)$, and hypotension. Two patients passed away post-procedure.

Median procedure time was 41 minutes (range 16-121 min), with the 41 -minute time occurring during the 19th procedure. Procedure durations further reduced, with the last 10 procedures being 20 minutes or under (nonlinear regression $\mathrm{p}$ value $P<0.0001$ ).

Conclusion Endoscopists experienced in EUS-GBD are expected to achieve a reduction in procedure time over successive cases, with efficiency reached at 41 minutes and a learning rate of 19 cases. Continued improvement is demonstrated with additional experience

\section{Introduction}

Surgery remains the gold standard for treatment of acute cholecystitis. However, in poor operative candidates, nonsurgical measures are required to prevent complications. Nonsurgical gallbladder decompression is traditionally achieved through percutaneous gallbladder drainage (PTGBD). However, PTGBD has been associated with significant complications including bile leak, peritonitis, bowel perforation, and catheter dislodge- ment [ 1 -5] as well as poor quality of life. In the past decade, endoscopic ultrasound-guided gallbladder drainage (EUSGBD) has emerged as an efficacious, safe alternative to PTGBD for patients with cholecystitis who cannot undergo cholecystectomy [6-12]. The procedure involves identifying and accessing the gallbladder using EUS, creating a fistulous tract, and deploying a stent across the tract [13]. Different techniques have been used for EUS-GLB as technology and operator experience improved [14-20]. However, it remains a technically chal- 
lenging procedure, requiring skills in EUS, fluoroscopy, and stent deployment. The learning curve of EUS-GBD is unknown. The aim of this study was to define the learning curve for EUSGBD.

\section{Patients and methods}

\section{Study overview}

Consecutive patients undergoing EUS-GLB by a single operator were included from a prospective registry over 5 years. All patients who underwent EUS-GBD were included. Patients underwent EUS-GBD drainage if they were diagnosed with cholecystitis and deemed unfit for surgical cholecystectomy. Demographics, procedure information, post-procedure follow-up data, and information on adverse events (AE) were collected. Non-linear regression and CUSUM analyses were conducted for the learning curve.

Technical success was defined as successful placement of a decompressing stent into the gallbladder. Clinical success was defined as resolution of cholecystitis post-procedure. AEs were graded according to the American Society for Gastrointestinal Endoscopy lexicon severity grading system. All patients were followed while inpatients until resolution of cholecystitis system. When feasible, patients were followed in the outpatient office 4 to 6 weeks post-procedure and every 6 months thereafter or earlier if recurrent symptoms developed.

\section{Procedural technique}

All procedures were performed under general anesthesia, by an endoscopist experienced in therapeutic EUS and ERCP (MK) (over 1000 cases at a center doing $>900$ therapeutic EUS per year). Patients were given antibiotics peri-procedurally if they were not already on antibiotic therapy.

An echoendoscope (GF-UCT 180, Olympus, Center Valley, Pennsylvania, United States) was advanced into the stomach or duodenum. The gallbladder was identified endosonographically. The gallbladder was accessed with a 19-gauge fine-needle aspiration needle after which a 0.035 -inch guidewire was advanced through the needle into the gallbladder lumen. The needle was removed, and the fistulous tract was created with either a dilating balloon with/without a needle-knife cautery catheter or a cautery-enhanced stent delivery system. In some cases, the gallbladder was accessed directly with a cautery-enhanced stent delivery system without preceding wire access. A lumen-apposing metal stent (LAMS) (Axios, Boston Scientific, Natick, Massachusetts, United States), fully-covered self-expanding metal biliary stent (FCSEMS) (Viabil, Conmed, Utica, New York, United States) or plastic stent was then deployed with one end in the gallbladder lumen and the other end in the stomach or duodenum. If a LAMS was used, the lumen of the stent was dilated to the diameter of the stent using a dilating balloon. In cases where the stent was misdeployed with one flange outside of the desired lumen, a bridging stent was placed through the misdeployed stent as previously described by Tyberg et al. [21, 22].
Patients were monitored overnight post-procedure. Clear liquid diet was initiated as early as post-procedure day 1 when clinical course allowed.

\section{Statistical analysis}

Consecutive patients undergoing EUS-GBD drainage by a single operator were included for statistical analyses from a prospective registry over 5 years. Demographics, procedure information, post-procedure follow-up data, and information on AEs were collected. Non-linear regression and CUSUM analyses were conducted for the learning curve using Stata 15 (College Station, Texas). Clinical success was defined as resolution of cholecystitis post-procedure.

\section{Results}

Forty-eight patients were included (58\% male, mean age 76 ). Twenty patients ( $42 \%$ ) had malignant cholecystitis, the remainder had benign disease. Technical success was 100\%. Most patients had LAMS placed $(15 \mathrm{~mm}, \mathrm{n}=29,60 \% ; 10 \mathrm{~mm}, \mathrm{n}=8$, $17 \%$; total $n=37,77 \%), 25$ of which were cautery-enhanced. The remaining patients had FCSEMS $(n=9,19 \%)$ or plastic stents alone $(n=2,4 \%)$. One patient required placement of a bridging stent to achieve technical success. The majority of stents were transduodenal $(n=28,58 \%)$, the remaining transgastric $(n=15,31 \%)$ or transjejunal $(n=4,8 \%)$. One patient had two stents placed from transgastric and transduodenal position during the same procedure. $>$ Table 1 summarizes characteristics of and clinical data on 48 patients who underwent EUS-GLB for management of cholecystitis.

Clinical success was achieved in 35 patients ( $72 \%)$. Of the remaining 13 patients, seven were lost to follow-up. Four patients had persistent cholecystitis symptoms, all of whom passed away from comorbidities of metastatic cancer or cardiac disease. Two patients passed away post-procedure prior to resolution of symptoms (see below). Mean follow-up time was 5.4 months (range $0-45$ ). During the follow-up period, six patients required reintervention for stent occlusion or migration with repeat endoscopy or surgical cholecystectomy. Two had their stents occluded with food/debris but had recovered enough to become surgical candidates and went for surgical cholecystectomy, three had their stents occluded with food/debris and required repeat stenting due to the obstruction, and one had a migrated stent that was replaced.

Nine patients (19\%) had AEs, seven moderate and two fatal. Four patients had post-procedure bleeding managed by repeat endoscopy with packing of plastic stents and in one patient embolization by interventional radiology. Two patients had liver abscesses managed with antibiotics $(n=1)$ or percutaneous drainage $(n=1)$ and one patient had hypotension immediately post-procedure requiring re-look endoscopy without additional intervention. Two post-procedure deaths occurred, one due to respiratory failure immediately post-extubation and one due to septic shock with peritonitis due to a perforated GB wall. That patient was subsequently diagnosed with gangrenous gallbladder. The AEs in our study occurred evenly throughout the learning curve. 
- Table 1 Patient characteristics.

\begin{tabular}{|c|c|}
\hline Characteristics & $n=48$ \\
\hline Age (mean) & 76.2 years ( 13.9 STD) \\
\hline Gender-male & $28 / 48(58 \%)$ \\
\hline \multicolumn{2}{|l|}{ Indication for EUS-GLB: } \\
\hline - Malignant cholecystitis & $20(42 \%)$ \\
\hline - Benign cholecystitis & $28(58 \%)$ \\
\hline Technical Success: & $48 / 48(100 \%)$ \\
\hline \multicolumn{2}{|l|}{ Approach: } \\
\hline - Transgastric & $15(31 \%)$ \\
\hline - Transduodenal & $28(58 \%)$ \\
\hline - Transjejunal & $4(8 \%)$ \\
\hline - Transgastric + transduodenal & $1(2 \%)$ \\
\hline \multicolumn{2}{|l|}{ Stent placement: } \\
\hline - LAMS- $15 \mathrm{~mm}$ & $29(60 \%)$ \\
\hline - LAMS $-10 \mathrm{~mm}$ & $8(17 \%)$ \\
\hline - Cautery-enhanced LAMS & $25(52 \%)$ \\
\hline - FCSEMS & $9(19 \%)$ \\
\hline - Plastic & $2(4 \%)$ \\
\hline - Bridging stents & $1(2 \%)$ \\
\hline Adverse events & $9 / 48(19 \%)$ \\
\hline - Bleeding & 4 \\
\hline - Hepatic abscess: & 2 \\
\hline - Hypotension in PACU & 1 \\
\hline - Peritonitis (death) & 1 \\
\hline - Respiratory failure (death) & 4 \\
\hline Clinical success & $35 / 48(72 \%)$ \\
\hline Procedure time (mins) & $41.1 \mathrm{~min}$ \\
\hline Reintervention required: & $6(12.5 \%)$ \\
\hline Mean follow-up time (months) & 5.4 \\
\hline $\begin{array}{l}\text { EUS-GLB, endoscopic ultrasound-gui } \\
\text { apposing metal stent; FCSEMS, fully- } \\
\text { fully covered self-expandable metal s }\end{array}$ & $\begin{array}{l}\text { Ider drainage; LAMS, lume } \\
\text { If-expanding metal stent } \\
\text { J, post-anesthesia care uni }\end{array}$ \\
\hline
\end{tabular}

Median procedure time was 41 minutes (range 16-121 min). The CUSUM chart ( Fig. 1 ) shows that the 41 -minute duration was achieved at the 19th procedure, hence indicating efficiency. Procedure durations further reduced with the last 10 procedures being 20 minutes or under (nonlinear regression pvalue: $P<0.0001$ ) ( $\triangleright$ Fig. 2 ) indicating continued improvement with experience.

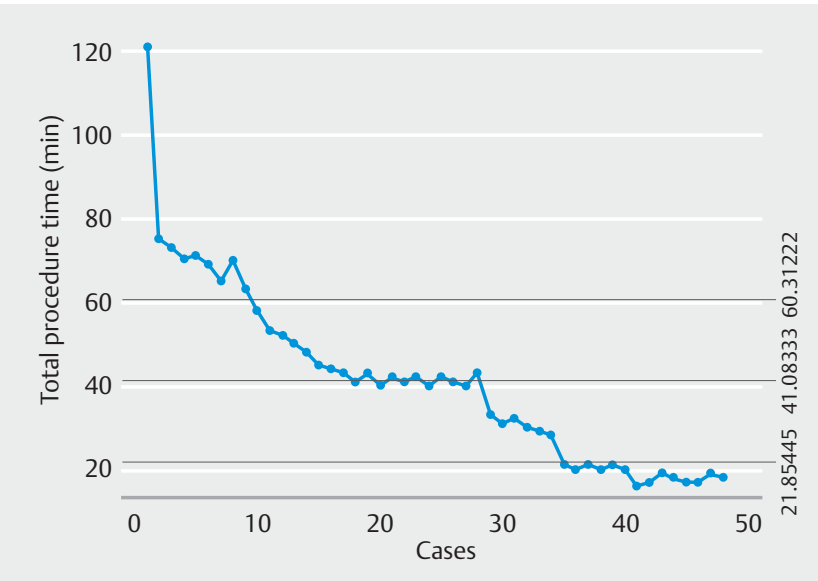

Fig. 1 The CUSUM chart.

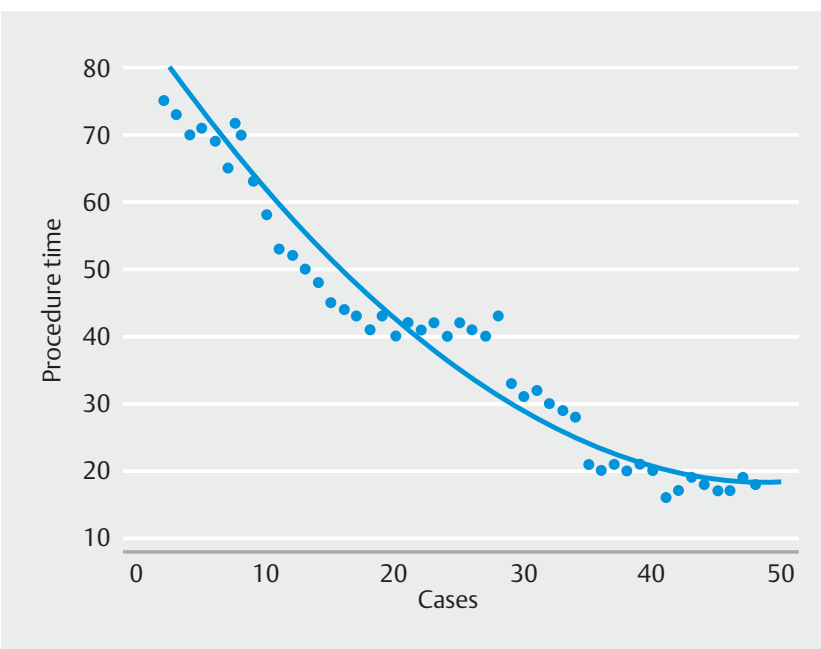

- Fig. 2 Nonlinear regression chart.

\section{Discussion}

EUS-GBD has emerged as a safe and efficacious option for patients who are unable to undergo surgical cholecystectomy. Published literature has demonstrated impressive safety and efficacy of the procedure $[6-8,10]$, as well as comparable and in some cases improved efficacy and safety over percutaneous cholecystostomy $[9,23]$. However, EUS-GBD is technically challenging and requires skill in multiple endoscopic modalities such as therapeutic EUS, wire manipulation, and stent deployment.

The learning curve for EUS-GBD remains unknown. Previous literature on a comparable procedure demonstrated that the number of AEs associated with EUS-guided biliary drainage is inversely proportional to the learning curve [24]. Similarly, Oh et al. noted that procedure times and AEs reduce after 24 cases and stabilized after 33 cases for endoscopic ultrasound-guided hepaticogastrostomy (EUS-HGS) [25]. Intuitively, increasing EUS-GBD experience could be expected to similarly correlate with decreased procedure times and improved safety. In our 
study, efficiency was reached at 41 minutes with a learning rate of 19 cases which is similar to aforementioned findings.

The AEs in our study occurred evenly throughout the learning curve. This could suggest that safety does not necessarily correlate with operator experience, but more likely is due to the fact that the AEs were more related to patients' underlying comorbidities than to operator performance. The two deaths seen in our study were the result of an anesthesia complication (mucous plugging post-extubation with subsequent respiratory arrest) and a perforated gallbladder that was present prior to endoscopic intervention.

Limitations of the study include the fact that all procedures were performed by a single operator with a large therapeutic EUS experience, meaning the results of this study are not applicable to endoscopists without the same level of experience. Moreover, additional learning curves from other expert endoscopists are needed to verify these findings. Another limitation is introduction of new technology throughout the study duration, most significantly introduction of the cautery-enhanced LAMS. This stent allowed for faster procedure times by negating the need for needle puncture and fistulous tract dilation and/or cautery with separate catheters.

\section{Conclusion}

In conclusion, defining the learning curve for novel therapeutic procedures is important. From our data, endoscopists experienced in therapeutic EUS can be expected to achieve a reduction in procedure time of EUS-GBD over successive cases, with efficiency reached at 41 minutes and a learning rate of 19 cases. Continued improvement was also noted with additional experience. AEs may be more related to patient comorbidities than procedural technique. Additional studies are required for further validation and exploration of these results.

\section{Competing interests}

Dr. Kahaleh has done consulting work for Boston Scientific, Interscope Med, and Abbvie. He has received research grants from Boston Scientific, Emcision, Conmed, Pinnacle, Cook, Gore, Merit, and Olympus. He is the CEO and Founder of Innovative Digestive Health Education \& Research Inc, Therapeutic Endoscopic Ultrasound Society and Obesity Coalition Inc. He is the CEO and Co-Founder of Clinical Research Directory Inc.

Dr. Gaidhane has done consulting work for Interscope Med. She is the COO of Therapeutic Endoscopic Ultrasound Society, Innovative Digestive Health Education \& Research Inc. \& Obesity Coalition. She is the Co-CEO of Clinical Research Directory Inc. Dr. Tyberg has done consulting work for NinePoint Medical, EndoGastric Solutions, and Obalon Therapeutics. She is a director at Therapeutic Endoscopic Ultrasound Society. Dr. Kedia is a Consultant for Boston Scientific.

\section{References}

[1] Akhan O, Akinci D, Ozmen MN. Percutaneous cholecystostomy. Eur J Radiol 2002; 43: 229-236

[2] Súbtil JC. Gallbladder drainage guided by endoscopic ultrasound. World J Gastrointest Endosc 2010; 2: 203-209

[3] Morse BC, Smith JB, LawdahI RB et al. Management of acute cholecystitis in critically ill patients: contemporary role for cholecystostomy and subsequent cholecystectomy. Am Surg 2010; 76: 708-712

[4] Hatzidakis AA, Prassopoulos P, Petinarakis I et al. Acute cholecystitis in high-risk patients: percutaneous cholecystostomy vs conservative treatment. Europ Radiol 2002; 12: 1778 - 1784

[5] Adler DG. Avoiding the tube: ERCP and EUS approaches to gallbladder drainage as alternatives to percutaneous cholecystostomy in patients with cholecystitis. Gastrointest Endosc 2019; 89: 299-300

[6] Minaga K, Yamashita Y, Ogura T et al. Clinical efficacy and safety of endoscopic ultrasound-guided gallbladder drainage replacement of percutaneous drainage: A multicenter retrospective study. Digest Endosc 2019; 31: $180-187$

[7] Saumoy M, Tyberg A, Brown E et al. Successful cholecystectomy after endoscopic ultrasound gallbladder drainage compared with percutaneous cholecystostomy, can it be done? J Clin Gastroenterol 2019; 53 : $231-235$

[8] Irani S, Ngamruengphong S, Teoh A et al. Similar efficacies of endoscopic ultrasound gallbladder drainage with a lumen-apposing metal stent versus percutaneous transhepatic gallbladder drainage for acute cholecystitis. Clin Gastroenterol Hepatol 2017; 15: 738 - 745

[9] Teoh A, Serna C, Penas I et al. Endoscopic ultrasound-guided gallbladder drainage reduces adverse events compared with percutaneous cholecystostomy in patients who are unfit for cholecystectomy. Endoscopy 2016; 49: $130-138$

[10] Tyberg A, Saumoy M, Sequeiros EV et al. EUS-guided Versus percutaneous gallbladder drainage: isn't it time to convert? J Clin Gastroenterol 2016; 52: 79-84

[11] Duncan CB, Riall TS. Evidence-based current surgical practice: calculous gallbladder disease. J Gastrointest Surg 2012; 16: 2011 - 2025

[12] Anderloni A, Buda A, Vieceli F et al. Endoscopic ultrasound-guided transmural stenting for gallbladder drainage in high-risk patients with acute cholecystitis: a systematic review and pooled analysis. Surg Endosc 2016; 30: $5200-5208$

[13] Irani S, Baron TH, Grimm IS et al. EUS-guided gallbladder drainage with a lumen-apposing metal stent (with video). Gastrointest Endosc 2015; 82: $1110-1115$

[14] Song T], Park DH, Eum JB et al. EUS-guided cholecystoenterostomy with single-step placement of a $7 \mathrm{~F}$ double-pigtail plastic stent in patients who are unsuitable for cholecystectomy: a pilot study (with video). Gastrointest Endosc 2010; 71: 634-640

[15] Widmer J, Alvarez P, Gaidhane M et al. Endoscopic ultrasonographyguided cholecystogastrostomy in patients with unresectable pancreatic cancer using anti-migratory metal stents: A new approach: EUSguided cholecystogastrostomy. Digest Endosc 2014; 26: 599-602

[16] de la Serna-Higuera C, Pérez-Miranda M, Gil-Simón P et al. EUS-guided transenteric gallbladder drainage with a new fistula-forming, lumenapposing metal stent. Gastrointest Endosc 2013; 77: 303 - 308

[17] Jang JW, Lee SS, Park DH et al. Feasibility and safety of EUS-guided transgastric/transduodenal gallbladder drainage with single-step placement of a modified covered self-expandable metal stent in patients unsuitable for cholecystectomy. Gastrointest Endosc 2011; 74: $176-181$

[18] Itoi T, Binmoeller KF, Shah J et al. Clinical evaluation of a novel lumenapposing metal stent for endosonography-guided pancreatic pseudocyst and gallbladder drainage (with videos). Gastrointest Endosc 2012; 75: 870-876 
[19] Moon JH, Choi H], Kim DC et al. A newly designed fully covered metal stent for lumen apposition in EUS-guided drainage and access: a feasibility study (with videos). Gastrointest Endosc 2014; 79: 990 - 995

[20] Teoh AYB, Binmoeller KF, Lau JYW. Single-step EUS-guided puncture and delivery of a lumen-apposing stent for gallbladder drainage using a novel cautery-tipped stent delivery system. Gastrointest Endosc 2014; 80: 1171

[21] Tyberg A, Saumoy M, Kahaleh M. Using NOTES to salvage a misdeployed lumen-apposing metal stent during an endoscopic ultrasound-guided gastroenterostomy. Endoscopy 2017; 49: 1007-1008
[22] Tyberg A, Zerbo S, Barthet M et al. A novel technique for salvaging a dislodged lumen-apposing metal stent during creation of an endoscopic gastrojejunostomy. Gastrointest Endosc 2016; 83: 254

[23] Yamashita Y, Takada T, Kawarada Y et al. Surgical treatment of patients with acute cholecystitis: Tokyo Guidelines. J Hepato-BiliaryPancr Surg 2007; 14: $91-97$

[24] Poincloux L, Rouquette O, Buc E et al. Endoscopic ultrasound-guided biliary drainage after failed ERCP: cumulative experience of 101 procedures at a single center. Endoscopy 2015; 47: $794-801$

[25] Oh D, Park DH, Song T] et al. Optimal biliary access point and learning curve for endoscopic ultrasound-guided hepaticogastrostomy with transmural stenting. Therap Adv Gastroenterol 2017; 10: 42 - 53 